\title{
Cited 2 controls left-right patterning and heart development through a Nodal-Pitx2c pathway
}

\author{
Simon D Bamforth ${ }^{1,6}$, José Bragança ${ }^{1,6}$, Cassandra R Farthing ${ }^{1,6}$, Jürgen E Schneider ${ }^{1}$, Carol Broadbent ${ }^{1}$, \\ Anna C Michell ${ }^{1}$, Kieran Clarke ${ }^{2}$, Stefan Neubauer ${ }^{1}$, Dominic Norris ${ }^{3}$, Nigel A Brown ${ }^{4}$, \\ Robert $\mathrm{H}$ Anderson ${ }^{5}$ \& Shoumo Bhattacharya ${ }^{1}$
}

Malformations of the septum, outflow tract and aortic arch are the most common congenital cardiovascular defects and occur in mice lacking Cited2, a transcriptional coactivator of TFAP2. Here we show that Cited $2^{-/}$mice also develop laterality defects, including right isomerism, abnormal cardiac looping and hyposplenia, which are suppressed on a mixed genetic background. Cited $2^{-/-}$mice lack expression of the Nodal target genes Pitx2c, Nodal and Ebaf in the left lateral plate mesoderm, where they are required for establishing laterality and cardiovascular development. CITED2 and TFAP2 were detected at the Pitx2c promoter in embryonic hearts, and they activate Pitx2c transcription in transient transfection assays. We propose that an abnormal Nodal-Pitx2c pathway represents a unifying mechanism for the cardiovascular malformations observed in Cited $2^{-/-}$mice, and that such malformations may be the sole manifestation of a laterality defect.

Genetic, developmental and molecular studies over the past decade have identified a number of DNA-binding transcription factors that have key roles in cardiac morphogenesis and in the pathogenesis of common congenital heart defects ${ }^{1}$. The role of transcriptional coactivators, molecules that connect DNA-binding transcription factors to the core transcriptional machinery, in cardiac development has only recently become apparent. These coactivators are exemplified by the paralogous genes EP300 and CREBBP ${ }^{2}$. Mutations in CREBBP cause Rubinstein-Taybi Syndrome ${ }^{3}$ and are frequently associated with cardiac malformations ${ }^{4}$.

EP300 and CREBBP interact with high affinity with a ubiquitously expressed cytokine and hypoxia-inducible transcriptional coactivator called CITED2 (also called p35srj and Mrg1) ${ }^{5-8}$. Binding of CITED2 to EP300 competitively inhibits the binding of the transcription factor HIF1A to EP300, blocking hypoxia-activated gene transcription ${ }^{5,8}$. Cited2 is essential for normal development of the heart, adrenals and nervous system ${ }^{9-13}$ and for fibroblast proliferation ${ }^{14}$. Mice lacking Cited2 die prenatally with diverse cardiovascular malformations, including atrial and ventricular septal defects, double-outlet right ventricle, common arterial trunk and aberrant aortic arches.

In addition to functioning as a transcriptional repressor of HIF1A, CITED2 also physically interacts with and coactivates TFAP2 (transcription factor AP2, also called Tcfap2) and LIM-domain containing transcription factors by linking them to EP300 and CREBBP $9,15,16$. Mutations in Tcfap $2 a$ and TFAP2B (Char syndrome) result in cardiac and aortic arch malformations ${ }^{17,18}$, suggesting that coactivation of TFAP2 by CITED2, EP300 and CREBBP is necessary for the normal development of these structures ${ }^{9}$. An alternative explanation for the development of cardiac malformations in mice lacking Cited 2 is dysregulation of hypoxia-activated gene transcription ${ }^{12}$.

The cardiovascular malformations resulting from deficiency of Cited2 encompass a diverse and variable spectrum that is not explained by effects on a single developmental process. For instance, although the deficiency of neural crest cells expressing Erbb3 in Cited $2^{-1-}$ embryos explains abnormal aortic arch remodeling ${ }^{9}$, it does not explain the atrioventricular septal defects. Cited 2 may be independently required for different aspects of cardiovascular development, with phenotypic variability resulting from random segregation of genetic modifiers. To test this idea, we characterized the cardiac phenotypes of coisogenic C57BL/6J Cited $2^{-1-}$ embryos. We found that the C57BL/6J background had a marked effect on the phenotype of Cited $2^{-1-}$ mice, resulting in left-right patterning defects. These results indicate that Cited 2 has a previously unsuspected role in establishing embryonic laterality and provide a unifying mechanism that explains the development of the diverse cardiovascular malformations in mice lacking Cited2.

\footnotetext{
${ }^{1}$ Department of Cardiovascular Medicine, University of Oxford, Wellcome Trust Centre for Human Genetics, Roosevelt Drive, Oxford OX3 7BN, UK.

${ }^{2}$ Department of Physiology, University of Oxford, South Parks Road, Oxford OX1 3QU, UK. ${ }^{3}$ MRC Mammalian Genetics Unit, Harwell OX11 ORD, UK. ${ }^{4}$ St. George's Hospital Medical School, University of London, Cranmer Terrace, London SW17 ORE, UK. ${ }^{5}$ Cardiac Unit, Institute of Child Health, University College London, 30 Guilford Street, London WC1N 1EH, UK. ${ }^{6}$ These authors contributed equally to this work. Correspondence should be addressed to S.B.

(shoumo.bhattacharya@well.ox.ac.uk).
}

Published online 10 October 2004; doi:10.1038/ng1446 
Table 1 Left-right patterning and cardiac defects in Cited2-lembryos

C57BL/6J Mixed background

Cardiovascular defects:

Right atrial isomerism

Common atrioventricular valve

$7 / 11$

$5 / 11$

$7 / 11$

Cilateral inferior caval vein

$0 / 11$

$1 / 11$

$5 / 11$

$7 / 11$

$0 / 11$

$10 / 11$

$10 / 11$

2/11

$2 / 11$

$4 / 11$

$3 / 15$

$3 / 15$

$2 / 15$

$1 / 15$

$1 / 15$

$2 / 15$

$2 / 15$

Ostium primum atrial septal defect*

Ventricular septal defect

Common arterial trunk

Interrupted fourth aortic arch

Right aortic arch

$3 / 15$

$10 / 15$

$7 / 15$

$1 / 15$

$2 / 15$

$4 / 15$

Noncardiac left-right patterning defects:

Right pulmonary isomerism

Small or rudimentary spleen

Visceral situs inversus

Tail on left, Cited2-1-

Tail on left, Cited2 ${ }^{+/+}$

*Ostium primum atrial septal defect without right atrial isomerism.

\section{RESULTS}

\section{Left-right patterning defects in Cited2 $2^{-/}$embryos}

We characterized Cited $2^{-1-}$ embryos on mixed $(129$ Ola $\times$ C57BL6/J) and coisogenic C57BL/6J backgrounds using magnetic resonance imaging (MRI). In addition to the heart and aortic arch defects previously reported ${ }^{9,11,12}$, these embryos had abnormal left-right patterning (Table 1). The left-right patterning abnormalities were significantly more frequent in coisogenic C57BL/6J (7 of 11) than in mixed-background ( 3 of 15$)$ Cited $2^{-1-}$ embryos $\left(\chi^{2}=5.1, P \leq 0.025\right)$. The abnormalities included right atrial isomerism (Fig. 1c,d), cardiac dextroposition (Fig. 1c), bilateral or midline inferior caval vein (Fig. 1d), abnormalities in ventricular topology (Fig. 1g-j), right pulmonary isomerism (Fig. 2b,d), small or rudimentary spleens (Fig. 2f), and abnormal embryonic turning (assessed at 9.5-10.5 d post coitum (d.p.c.), Fig. 2j). In 1 Cited $2^{-l-}$ embryo (of 26 studied by MRI) we observed abdominal visceral situs inversus (Fig. 2h). These malformations were not observed in wild-type embryos from either genetic background (Figs. 1a,b,e,f and 2a,c,e,g,i). We used Hand1 (also called $e H a n d)$ riboprobes to mark the left ventricle ${ }^{19}$ and found that it was positioned either posterior or dextral to the right ventricle in $\mathrm{C} 57 \mathrm{BL} / 6 \mathrm{~J}$ Cited $2^{-1-}$ embryos, confirming that they had ventrally or sinistrally looped hearts (Fig. 3b,d; compare with wild-type hearts in Fig. 3a,c).

\section{Pitx2c is deficient in C57BL6/J Cited2 ${ }^{-/-}$embryos}

A key feature of the phenotype of C57BL6/J Cited2 ${ }^{-/-}$embryos, right isomerism and hyposplenia, also occurs in mice lacking Pitx2, a homeobox transcription factor ${ }^{20-23}$. This left-determining activity specifically resides in the Pitx2c isoform, which is expressed asymmetrically in the left lateral plate mesoderm at 8.5 d.p.c. and later in the developing heart, lungs and gut ${ }^{21,24}$. Consistent with previous observations $^{21}$, we detected Pitx2 asymmetrically in the left lateral plate mesoderm and symmetrically in the head mesoderm in wild-type embryos at 8.5 d.p.c. (Fig. 3e,f). In C57BL6/J Cited2 ${ }^{-1-}$ embryos, Pitx2 expression in the left lateral plate mesoderm was typically lost (in 9 of 11 embryos) whereas expression in the head mesoderm was normal, indicative of a specific deficiency of Pitx2c (Fig. 3g,h). Consistent with previous observations ${ }^{21,25}$, Pitx $2 c$ was expressed over the outflow tract and ventral surface of the right ventricle and in the left atrium in hearts of wild-type embryos (Fig. 3i,j) but was deficient in hearts of C57BL6/J Cited $2^{-l-}$ embryos (three of three; Fig. 3k,l).

\section{Effect of genetic background on Pitx2c expression}

To investigate the relative paucity of right isomerism in the mixed genetic background, we crossed coisogenic C57BL6/J Cited $2^{+/-}$and coisogenic $129 \mathrm{~Sv}$ Cited $2^{+/-}$mice. Pitx $2 c$ expression in the left lateral plate mesoderm of mixed-background Cited $2^{-1-}$ embryos $\left(F_{1}\right.$ generation, 8.5 d.p.c.) resembled that of the wild-type embryos (11 of 11; Fig. $3 \mathbf{m}-\mathbf{p}$ ), consistent with the reduced frequency of isomerism observed in the mixed background. Pitx $2 c$ expression in hearts of mixed-background $\mathrm{F}_{1}$ Cited $2^{-/}$embryos resembled that of the wildtype embryos in two of three cases (Fig. 3q,r). In the third case, Pitx $2 c$ expression was reduced in the outflow tract and right ventricle but preserved in the left atrium (Fig. 3s,t). Examination of mixed-background $\mathrm{F}_{1}$ Cited $2^{-l-}$ embryos at later stages by MRI indicated that they have cardiac abnormalities, such as ventricular septal defects and double-outlet right ventricle, but not laterality defects (three of three embryos; data not shown). The mixed-background $\mathrm{F}_{1}$ Cited2 $2^{-1-}$ embryos (seven of seven) turned normally (data not shown).

\section{Cardiac and laterality defects in Pitx $2 \mathrm{c}^{-1-}$ embryos}

To determine the extent to which the C57BL6/J Cited $2^{-1-}$ phenotype can be explained by Pit $2 c$ deficiency, we examined Pit $2 c^{-1-}$ embryos, which specifically lack the Pitx $2 c$ isoform ${ }^{25}$ and have defects in the aortic arch vessels, such as double-outlet right ventricle and right-sided or double aortic arches ${ }^{25}$. We found that 11 of 18 Pit $x 2 c^{-1-}$ embryos had bilateral, left-sided or midline inferior caval veins (Fig. 4a), and 16 of 18 embryos had right atrial and pulmonary isomerism (Fig. $\mathbf{4 b}, \mathbf{c}$ ). Although none had abnormal ventricular topology, 7 of 18 embryos had malposition of the heart to the right (Fig. 4d), and the hearts of the remaining embryos were in the midline rather than to the left. In addition, 16 of 18 embryos had ventricular septal defects with associated double-outlet right ventricle (Fig. 4e), and 3 of 18 embryos had right-sided aortic arches. Notably, we observed that 2 of 18 Pitx $2 c^{-l-}$ embryos had right-sided stomachs (Fig. 4f), and 1 of 18 had a midline stomach. The spleen was absent or small in 16 of 18 embryos. Unlike mice lacking all Pitx2 isoforms ${ }^{21,22}$, none of the Pitx $2 c^{-1-}$ embryos had defects in ventral body wall closure (Fig. 4d,f).

\section{Cited 2 is required for Nodal-activated transcription}

The asymmetric expression of Pitx $2 c$ in the left lateral plate mesoderm is controlled by Nodal, a TGF- $\beta$-like molecule. Nodal is expressed in the node and induces the asymmetric expression of Pitx $2 c$, Ebaf (also called Lefty2) and Nodal in the left lateral plate mesoderm ${ }^{26,27}$. Nodal was absent in the left lateral plate mesoderm (in 7 of 8 embryos) but present in the node of C57BL6/J Cited $2^{-1-}$ embryos, though at lower levels than in wild-type embryos (Fig. 5a-d). Ebaf, which functions as a Nodal antagonist $\mathrm{t}^{28,29}$ and is normally expressed in the left lateral plate mesoderm, was also absent in C57BL6/J Cited2 ${ }^{-1-}$ embryos (six of six; Fig. 5e,f). $C f c 1$ (also called Cryptic), a coreceptor for Nodal, was, in keeping with previous observations ${ }^{30}$, expressed in the lateral plate mesoderm in wild-type and C57BL6/J Cited $2^{-1-}$ embryos (two of two; Fig. 5g,h). Leftb (also called Lefty1), a Nodal antagonist that is normally expressed on the left side of the prospective floorplate in response to $\mathrm{Nodal}^{31}$, was restricted to the posterior floorplate in C57BL6/J Cited2 ${ }^{-1-}$ embryos (two of two; Fig. 5i-l). 

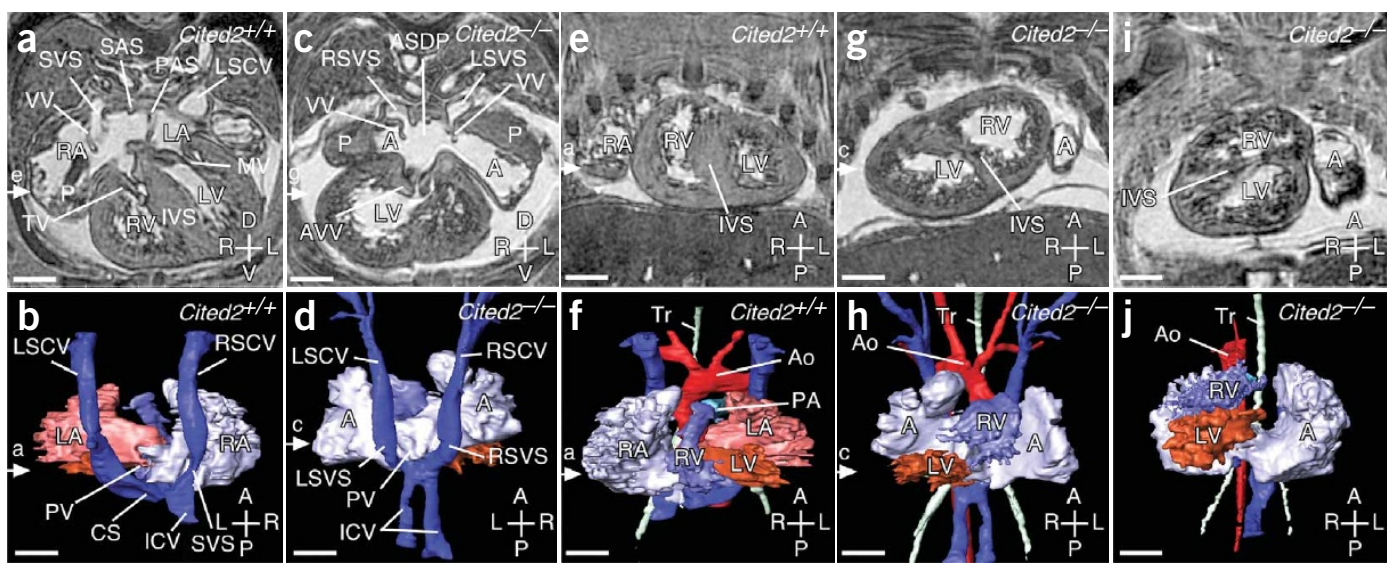

Figure 1 Cardiovascular laterality defects in Cited ${ }^{-1-}$ embryos at 15.5 d.p.c. (MRI of cardiac anatomy). (a-d) Transverse sections and three-dimensional reconstructions (dorsal views) of wild-type and $C$ ited $2^{-1-}$ embryonic hearts. (a,b) Wild-type heart showing a pectinated (P) right atrium (RA), with venous valves (VV) at the entrance of the systemic venous sinus (SVS), into which drain the right superior caval vein (RSCV), the left superior caval vein (LSCV; through the coronary sinus; CS) and the inferior caval vein (ICV). The left atrium (LA) is characterized by the primary atrial septum (PAS) and pulmonary venous drainage (PV). Other structures seen are secondary interatrial septa (SAS), right and left ventricles (RV, LV), interventricular septum (IVS) and mitral and tricuspid valves (MV, TV). (c,d) Cited $2^{-1-}$ heart showing a large primum atrial septal defect (ASDP), resulting in a common atrium (A). This is pectinated on each side and has bilateral systemic venous sinuses (LSVS, RSVS), into which drain the bilateral superior and inferior caval veins. A common

atrioventricular valve (AVV) opens into the left ventricle. These appearances are consistent with right atrial isomerism, where there is a failure to develop the intrinsic 'leftness' of the left-sided atrial chamber ${ }^{47}$. The heart is malpositioned to the right. (e-j) Coronal sections and three-dimensional reconstructions (ventral views) of wild-type and Cited $2^{-1-}$ embryonic hearts. (e,f) Wild-type heart where the right ventricle is dextral to the left and gives rise to the main pulmonary artery (PA). The left ventricle gives rise to the aorta (Ao). Also indicated is the trachea (Tr). (g,h) Cited2 ${ }^{-1-}$ heart with sinistral looping: the right ventricle is sinistral (and anterior) to the left ventricle. (i,j) Cited $2^{-1-}$ heart with ventral looping: the right ventricle is anterior to the left ventricle. Scale bars, $500 \mu \mathrm{m}$. Axes: D, dorsal; V, ventral; R, right; L, left; A, anterior; P, posterior. Arrows in each image indicate the other section planes.

\section{TFAP2 and CITED2 interact with the Pitx2c P1 promoter}

(2) To further examine the deficiency of Nodal-activated gene transcrip\pm tion in C57BL6/J Cited $2^{-1-}$ mice, we focused on Pitx2c. Pitx2c Z expression during development is controlled by an asymmetric E enhancer (ASE) and a P1 promoter ${ }^{32}$ (Fig. 6). Because one mechan을 ism for CITED2 function is coactivation of TFAP2, we used the (2) transcription factor database ${ }^{33}$ to identify putative TFAP2 binding sites in the mouse and human promoters ${ }^{32}$ (Fig. 6b). Alignment of the mouse and human Pitx2c P1 promoters also showed that they are extensively conserved (Fig. 6b). Using RT-PCR, we found that Tcfap2 isoforms, Cited 2 and Pitx $2 c$ were coexpressed in $\mathrm{C} 2 \mathrm{C} 12$ cells and in the embryonic heart (Supplementary Fig. 1 online). To determine whether endogenous CITED2 and TFAP2 physically interact with the Pitx2c P1 promoter and the ASE, we carried out chromatin
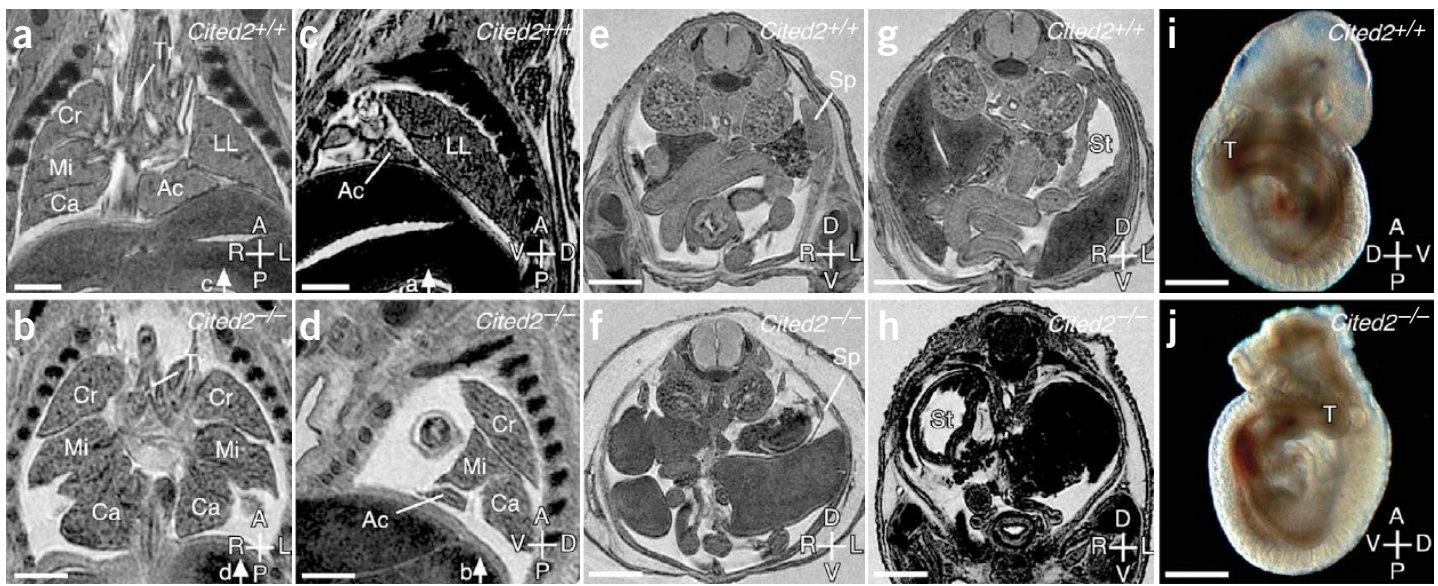

Figure 2 Noncardiovascular laterality defects in Cited2-l- embryos. (a-h) MRI of 15.5 d.p.c. embryos. (a,c) Coronal and sagittal sections of a wild-type embryo showing the left lung (LL); the cranial (Cr), caudal (Ca), middle (Mi) and accessory (Ac) lobes of the right lung; and the trachea (Tr).

(b,d) Corresponding sections through a Cited $2^{-1-}$ embryo showing that both lungs have four lobes, indicative of right pulmonary isomerism. (e) Transverse section of a wild-type embryo just posterior to stomach showing the spleen (Sp). (f) Corresponding section of a Cited2 ${ }^{-1-}$ embryo showing a rudimentary spleen. (g) Transverse section of a wild-type embryo showing a normal left-sided stomach (St). (h) Corresponding section of a Cited $2^{-1-}$ embryo showing a right-sided stomach. (i) Right lateral view of a Cited $2^{+++}$embryo showing that the tail $(\mathrm{T})$ is on the right, indicative of normal embryonic turning48. (j) Left lateral view of a Cited2-l- embryo showing the tail on the left. Scale bars, $500 \mu \mathrm{m}$. Axes: D, dorsal; V, ventral; R, right; L, left; A, anterior; P, posterior. Arrows in each image indicate the other section planes. 


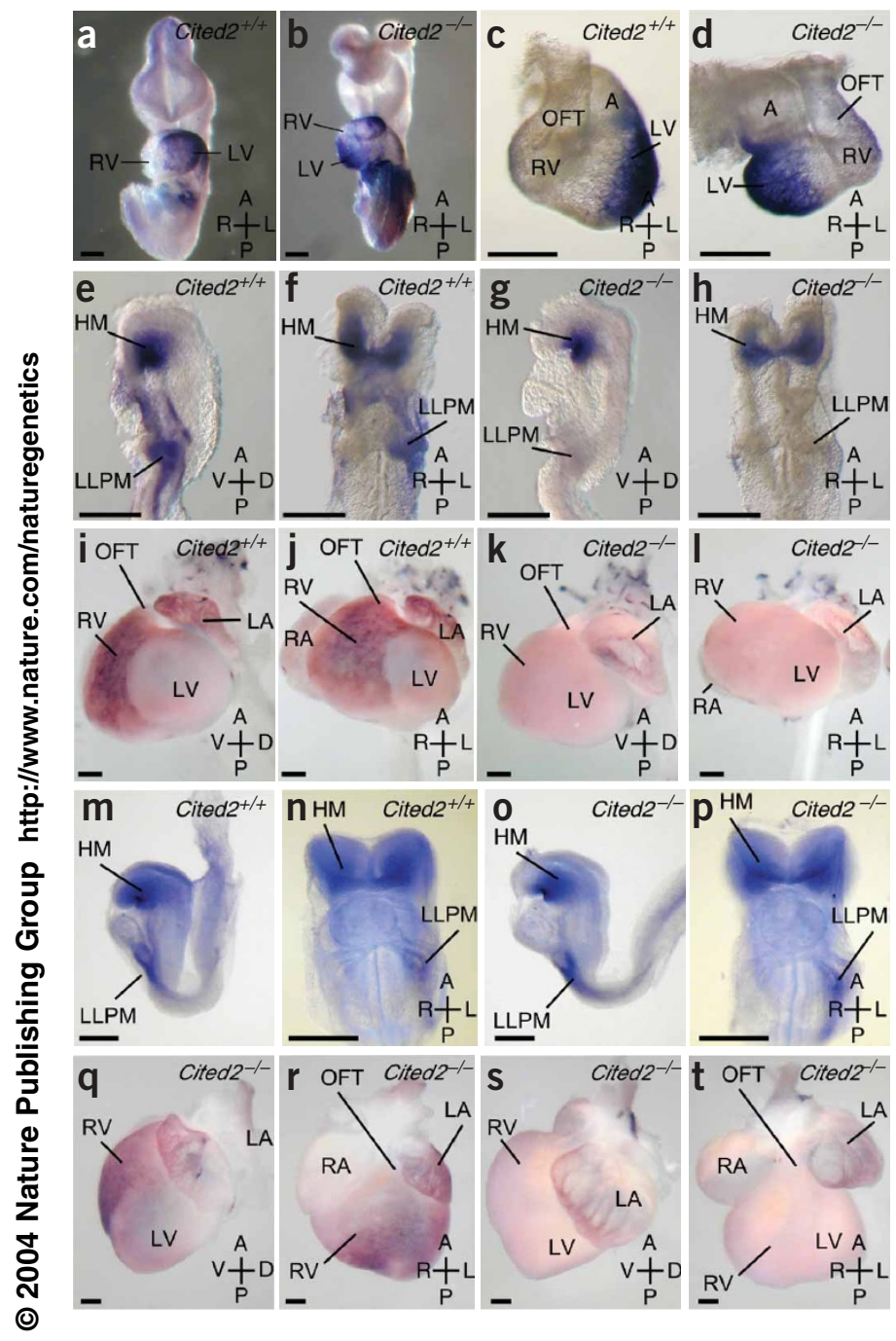

immunoprecipitation (ChIP) assays, initially from C2C12 cells. We reproducibly detected specific ChIP signals with antibodies specific to TFAP2A, TFAP2C and CITED2 (Fig. 7a). We were unable to detect ChIP signals using primers in the ASE (data not shown). We next carried out ChIP assays from wild-type embryonic hearts at 13.5 d.p.c. We detected specific and reproducible signals (in three independent experiments) with antibodies specific to acetylated histone $\mathrm{H} 3$, TFAP2A and CITED2 (Fig. 7b).

Figure 4 Cardiac and laterality defects in mice lacking Pitx2c. MRI showing sections of Pitx $2 c^{-1-}$ embryos at 15.5 d.p.c. (a) Transverse abdominal section showing bilateral inferior caval veins (RICV and LICV). The liver (Li) is indicated. (b) Transverse section through the heart showing right atrial isomerism, as indicated by the large primum atrial septal defect (ASDP), common atrium (A) and bilateral systemic venous sinuses (LSVS, RSVS). The right and left ventricles (RV, LV) and the interventricular septum (IVS) are indicated. (c) Sagittal section showing that the left-sided lung has four lobes (cranial ( $\mathrm{Cr}$ ), caudal (Ca), middle (Mi) and accessory (Ac)), indicative of right pulmonary isomerism. (d) Transverse thoracic section showing a right-sided (dextroposed) heart. The topology of the ventricles is normal: the left ventricle (LV) is sinistral to the right ventricle (RV). (e) Transverse section through the heart showing a ventricular septal defect (VSD).

(f) Transverse abdominal section showing a right-sided stomach (St). Scale bars, $500 \mu \mathrm{m}$. Axes: D, dorsal; V, ventral; R, right; L, left; A, anterior; $\mathrm{P}$, posterior.
Figure 3 Hand1 and Pitx2 expression in Cited2-l- embryos. (a-d) In situ hybridization of embryos and hearts at 9.5 d.p.c. with an antisense Hand1 riboprobe to mark the morphological left ventricle. (a) Ventral view of a Cited $2^{+++}$embryo showing a normal dextrally looped heart with a sinistral left ventricle. (b) Corresponding view of a Cited $2^{-1-}$ embryo showing a ventrally looped heart: the left ventricle is posterior to the right ventricle. (c) Ventral view of a Cited $2^{+/+}$heart showing the sinistrally positioned left ventricle. The outflow tract (OFT) and the atrium (A) are indicated. (d) Corresponding view of a sinistrally looped Cited $2^{-1-}$ heart with a dextral left ventricle. (e-p) In situ hybridization of stage-matched embryos at 8.5 d.p.c. and hearts at 15.5 d.p.c. with an antisense Pitx2 riboprobe. $\left(\right.$ e,f) $\mathrm{C} 57 \mathrm{BL} / 6 \mathrm{~J}$ Cited $^{+/+}$embryo showing left lateral and ventral views. Pitx2 isoforms are expressed symmetrically in the head mesoderm (HM), whereas Pitx2c alone is expressed asymmetrically in the left lateral plate mesoderm (LLPM). (g,h) Corresponding views of a C57BL/6J Cited $2^{-1-}$ embryo showing Pitx2 expression only in the head mesoderm (HM). (i,j) Left lateral and ventral views of a C57BL/6J Cited $2^{+/+}$heart. Pitx2c expression is detected in the ventral aspects of the outflow tract region (OFT) and of the right ventricle (RV) and also in the left atrium (LA). (k,I) Corresponding views of a C57BL/6J Cited2-l- heart showing absence of Pitx2c. Also indicated are the left ventricle (LV) and the right atrium (RA). (m,n) Left lateral and ventral views of a mixed-background $F_{1}$ Cited2 ${ }^{+/+}$embryo showing normal Pitx2c expression in the left lateral plate mesoderm (LLPM).

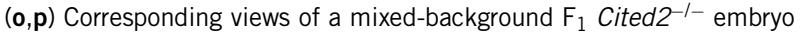
showing normal Pitx2c expression in the left lateral plate mesoderm (LLPM). $(\mathbf{q}, \mathbf{r})$ Left lateral and ventral views of a mixed-background $\mathrm{F}_{1}$ Cited $2^{-1-}$ heart showing normal Pitx2c expression. (s,t) Corresponding views of a mixedbackground $F_{1}$ Cited $2^{-1-}$ heart from a littermate embryo, showing markedly reduced Pitx2c expression in the right ventricle (RV) and the outflow tract (OFT), but normal expression in the left atrium (LA). Scale bars, $250 \mu \mathrm{m}$. Axes: D, dorsal; V, ventral; R, right; L, left; $A$, anterior; P, posterior.

\section{TFAP2 and CITED2 activate the Pitx2c P1 promoter}

To determine whether TFAP2 and CITED2 could directly activate the Pitx2c P1 promoter, we constructed a luciferase reporter vector (P1-Luc-ASE) that maintains the relative arrangement of the P1 and the ASE elements with respect to the transcriptional start site ${ }^{32}$. We also constructed a reporter vector that has P1 but lacks the ASE (P1-Luc), carried out transient transfection experiments in Hep3B cells and measured luciferase reporter activity. P1-Luc activity was not affected by CITED2 alone (Fig. 7c) but was activated by TFAP2A (by a factor of 1.9) and by TFAP2C (by a factor of 2.7). P1-Luc activity was further activated by TFAP2A plus CITED2 (by a factor of 2.6) and by TFAP2C plus CITED2 (by a factor of 3.5). The baseline reporter activity of P1-Luc-ASE was not affected by CITED2 alone. It was activated weakly by TFAP2A (by a factor of 1.2) and significantly more strongly by TFAP2A plus CITED2 (by a factor of 1.9). It was activated more strongly (by a factor of 2.0) by TFAP2C and, again, significantly

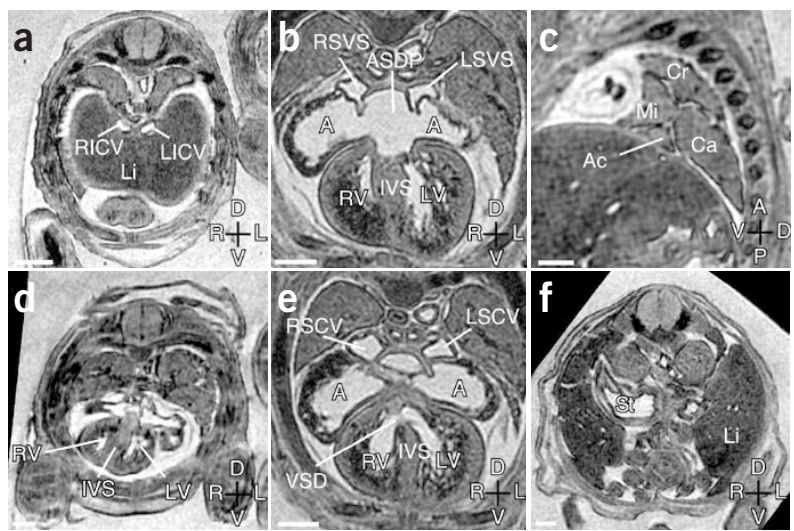


Figure 5 Cited2 is required for Nodal-activated gene transcription. In situ hybridization of stagematched C57BL6/J Cited $2^{+/+}$and C57BL6/J Cited $2^{-1-}$ embryos at $\sim 8.5$ d.p.c. with antisense riboprobes that detect Nodal, Ebaf, Cfcl and Leftb. $(\mathbf{a}, \mathbf{b})$ Ventral and left lateral views of a Cited $2^{+/+}$embryo showing normal expression of Nodal in the node and in the left lateral plate mesoderm (LLPM). (c,d) Corresponding view of a Cited $2^{-1-}$ embryo showing expression of Nodal in the node but not in the left lateral plate embryo showing normal expression of Ebaf in the left lateral plate mesoderm (LLPM).

(f) Corresponding view of a Cited $2^{-1-}$ embryo showing absence of Ebaf expression. (g,h) Ventral views of $\mathrm{Cited}^{+/+}$and Cited2 $2^{-1-}$ embryos, respectively, both showing normal expression of $C f c 1$ in the lateral plate mesoderm. (i,j) Ventral and left lateral views of a Cited $2^{+/+}$embryo showing normal expression of Leftb in the left prospective floorplate (LPFP). The arrowheads mark the extent of expression. (k,I) Corresponding views of a Cited $2^{-1-}$ embryo showing that Leftb expression is restricted to the posterior left prospective floorplate (LPFP). Scale bars, $250 \mu \mathrm{m}$. Axes: D, dorsal; V, ventral; R, right; L, left; A, anterior; P, posterior.
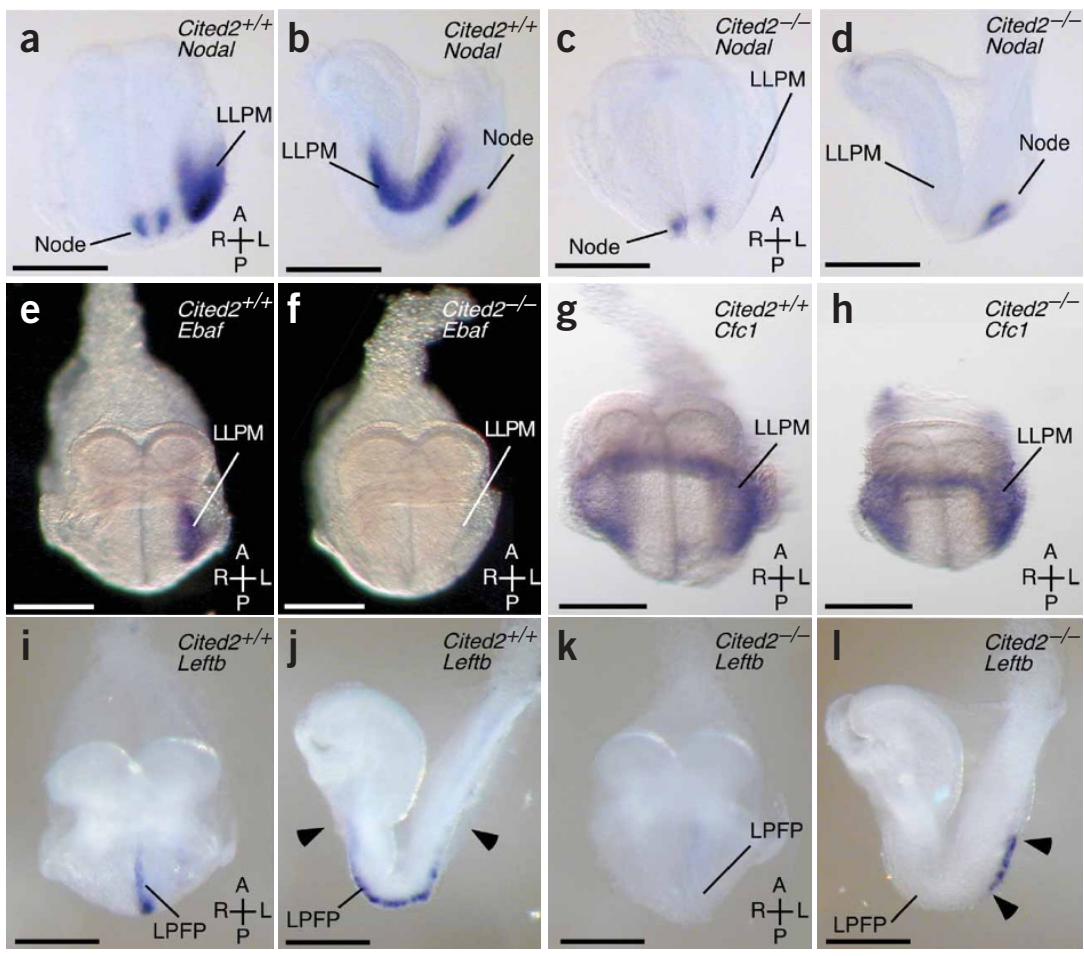

more strongly (by a factor of 3.1) by TFAP2C plus CITED2. Thus, TFAP2 isoforms and CITED2 can synergistically activate the Pitx2c P1-Luc-ASE reporter.

\section{TFAP2 binding sites in the Pitx2c P1 promoter}

To identify TFAP2 binding sites in the Pitx $2 c$ P1 promoter, we tested radioactively labeled double-stranded oligonucleotide probes (AB-WX; Fig. 6b) for binding to TFAP2A (generated by coupled in vitro transcription-translation using reticulocyte lysates) by electrophoretic mobility shift assay (EMSA). The addition of probes IJ, MN and QR to reticulocyte lysate containing TFAP2A resulted in the appearance of a specific band (Fig. 7d) that is competed out by a wild-type TFAP2 binding probe (MTIIa) but not by mutant or

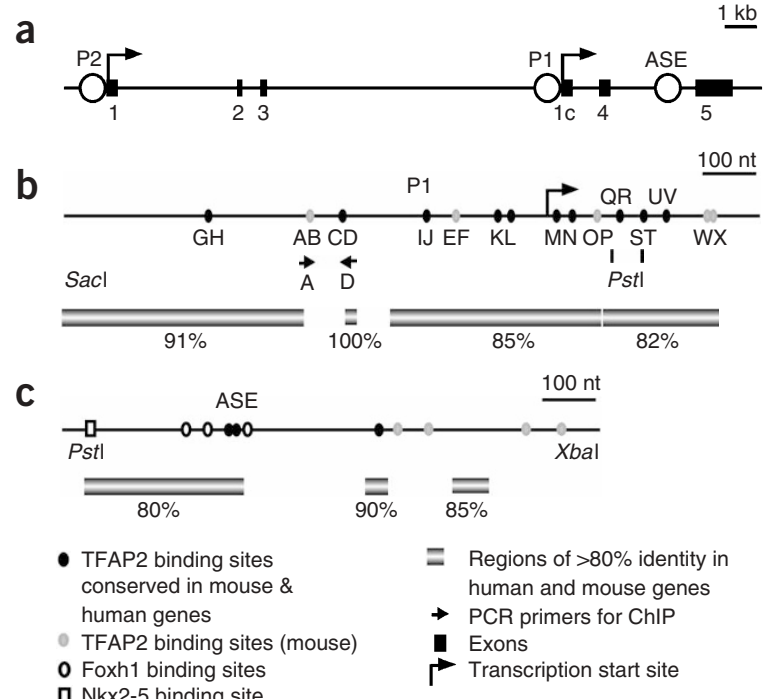

nonspecific probes (MTIIaMUT or PPAR). We obtained similar results for TFAP2C (data not shown). We next investigated the role of the TFAP2 binding sites IJ, $\mathrm{MN}$ and $\mathrm{QR}$ in promoter transcriptional activity by mutating them to prevent TFAP2 binding. Loss of TFAP2 binding by the mutant oligonucleotides was confirmed by EMSA (data not shown). We next introduced the IJ, MN and QR mutations in the Pitx2c P1 promoter by site-directed mutagenesis. The mutations were introduced in the $0.9-\mathrm{P} 1$ vector $^{32}$ and their effect examined in transient transfection experiments. The transcriptional activity of the construct containing the wildtype Pitx $2 c$ promoter $(0.9-\mathrm{P} 1)$ was significantly increased by the cotransfection of TFAP2 and CITED2. Mutations of the IJ, MN and QR TFAP2 binding sites (0.9-P1(IJMNQR)) led to significant decrease of reporter activation by TFAP2 isoforms in combination with CITED2 (Fig. 7e).

\section{DISCUSSION}

Our results, together with those reported previously ${ }^{9,11-13}$, show that on a mixed background, Cited $2^{-1-}$ embryos have a variable spectrum of cardiovascular malformations with defects in atrioventricular septation, outflow tract formation and aortic arch remodeling. Coisogenic

Figure 6 Evolutionary conservation of the Pitx2c P1 promoter. (a) Structure of the mouse Pitx 2 gene (after ref. 32). Splicing of exons 1c, 4 and 5 generates the Pitx2c isoform. The ASE in intron 5 and the P1 promoter in intron 3 are indicated. (b) The mouse P1 promoter contains multiple consensus TFAP2 binding sites, some of which are conserved in the human. The mouse and human $\mathrm{P} 1$ promoters also have extensive blocks of homology identified using BLAST ${ }^{44}$. The arrow indicates the transcriptional start site of exon 1c. The positions of PCR primers used for chromatin immunoprecipitation ( $A$ and $D$ ) and probes used for EMSA (AB-WX) are indicated. (c) The mouse Pitx2c ASE also contains three conserved TFAP2 binding sites and has small blocks of homology with the human ASE. Foxh1 and Nkx2-5 binding sites are indicated. 
Figure 7 CITED2 and TFAP2 control Pitx2c expression. (a) Left panel, ChIP from $\mathrm{C} 2 \mathrm{C} 12$ cells with the indicated polyclonal antibodies to TFAP2 isoforms, control preimmune serum and control polyclonal antibody to the Flag epitope. Immunoprecipitates were used in PCR with primers specific for the mouse Pitx $2 c$ promoter. Right panel, ChIP from $\mathrm{C} 2 \mathrm{C} 12$ cells using monoclonal antibody to CITED2 and to Stat1 (as control) or antibody to mouse IgG alone (No 1st $\mathrm{Ab})$. In the input lane, $12.5 \%$ of the chromatin

was used as PCR template. (b) ChIP from embryonic mouse hearts (13.5 d.p.c.) with the indicated antibodies as described above and with a polyclonal antibody to acetylated histone $\mathrm{H} 3$ (Ac-H3). Stat2-specific polyclonal antibody was used as control. In the input lane, $10 \%$ of the chromatin was used as PCR template. (c) Hep3B cells were transiently transfected with $40 \mathrm{ng}$ of the indicated plasmids and with CMV-lacZ (40 ng). Results (mean \pm s.e.m., four independent experiments) are presented as relative luciferase units (RLU), corrected for $\beta$-galactosidase activity. The control transfection value in each case (with CMV-vector) is set at 1 . ${ }^{*} P \leq 0.05 ;{ }^{*} P \leq 0.005$. (d) EMSA using Pitx2c P1 promoter-derived oligonucleotide probes IJ, MN and QR. Reticulocyte lysates containing TFAP2A were incubated with doublestranded radioactively labeled DNA probes and

O) 100-fold molar excess of competitors as indicated. Reticulocyte lysate programmed with pcDNA3 vector was used as control. The free probes are indicated by asterisks. The specific TFAP2A probe complex is indicated, as is a nonspecific
band (NS). IVTT, in vitro transcribed and translated peptide. (e) Hep3B cells were transiently transfected with 200 ng of 0.9-P1 or 0.9-P1(IJMNQR) independent experiments) are presented as relative lacZ units (RLacZU, corrected for luciferase activity). The control transfection value for each reporter (with CMV-vector) is set at $1 .{ }^{*} \mathrm{P} \leq 0.05$.

C57BL/6J Cited2 ${ }^{-1-}$ embryos also develop a spectrum of left-right (2) patterning abnormalities at a significantly high frequency, indicating that background-specific genetic modifiers have a key role in determining the phenotype. The appearance of diverse cardiac malformations in the mixed genetic background could then be a subtle manifestation of an underlying left-right patterning abnormality.

The left-right patterning defect in Cited $2^{-1-}$ embryos was characterized by right isomerism and hyposplenia, which are prominent features of Pitx2 deficiency ${ }^{20-24}$. Our results using Pitx $2 c^{-1-}$ mice definitively establish that these functions of Pitx2 reside in the asymmetrically expressed Pitx2c isoform. The loss of Pitx $2 c$ expression in C57BL/6J Cited2 $2^{-1-}$ embryos indicates that Cited2 functions genetically upstream of Pitx2c. Thus, the abnormal caval veins, right atrial and pulmonary isomerism, ventricular septal defects with double-outlet right ventricle, right-sided aortic arches, cardiac dextroposition, hyposplenia and occasional visceral situs inversus observed in C57BL/6J Cited $2^{-l-}$ embryos can be explained by Pitx2c deficiency. Notably, Pitx2c deficiency is not apparent in the left lateral plate mesoderm of mixed-background $\mathrm{F}_{1}$ Cited $2^{-l-}$ embryos, explaining the lack of overt laterality defects. But expression of Pitx2c in the hearts of mixed-background $\mathrm{F}_{1}$ Cited $2^{-1-}$ embryos can be abnormal, explaining the other cardiovascular malformations observed here. These results indicate that genetic modifiers of the Cited $2^{-1-}$ phenotype function by directly or indirectly controlling Pitx $2 c$ transcription.

The asymmetric expression of Pitx $2 c$ in the left lateral plate mesoderm is controlled by Nodal, a TGF- $\beta$-like molecule that is expressed in the node and then in the left lateral plate meso- derm $^{26,27,34}$. Nodal activity is thought to travel from the node to the left lateral plate mesoderm, and from the left lateral plate mesoderm to the midline ${ }^{31}$. The binding of Nodal to its receptor-coreceptor complex (consisting of Acvr2b, Acvrlb and Cfc1) results in phosphorylation of Smad2 (ref. 26). Phosphorylated Smad2 interacts with the transcription factor Foxh1 to activate target gene transcription through the asymmetric enhancers of Pitx2c, Nodal and Ebaf, thus mediating the Nodal-induced expression of these genes in the left lateral plate mesoderm ${ }^{29,31,32,35}$. Our data indicate that in C57BL/6J Cited $2^{-I-}$ embryos, although Nodal is expressed in the node, both Nodal and Ebaf are absent in the left lateral plate mesoderm. The absence of Pitx2c, Ebaf and Nodal in the left lateral plate mesoderm of C57BL/6J Cited $2^{-1-}$ embryos indicates that Cited2 is necessary for Nodal-activated gene transcription at this location.

Genetic evidence suggests that Cited2 functions, at least in part, downstream or in parallel to $C f c 1$. Deletion of $C f c l$ results in right isomerism, hyposplenism, abnormal cardiac looping and randomization of axial rotation and of visceral situs ${ }^{36,37}$. It also results in loss of Pitx2c, Ebaf and Nodal in the left lateral plate mesoderm and loss of Leftb in the left posterior floorplate, but with preservation of Nodal expression in the node ${ }^{36,37}$. C57BL/6J Cited $2^{-1-}$ embryos resemble those with $C f c 1$ deficiency, with three exceptions: (i) they do not have randomization of visceral situs; (ii) they have only partial loss of Leftb expression; and (iii) they express $C f c 1$ normally. Taken together, our data are most consistent with Cited 2 functioning, at least in part, downstream or in parallel to $C f c 1$ and upstream of Nodal, Pitx2c and Ebaf in the left lateral plate mesoderm. Notably, mutation causing 
near-complete loss of Nodal expression specifically in the left lateral plate mesoderm results in abnormal heart looping and loss of expression of Leftb and Ebaf, but only slightly delays and restricts expression of Pitx $2 c$ (ref. 35). Thus, the deficiency of Nodal expression in the left lateral plate mesoderm explains, at least in part, the abnormal looping and loss of Leftb and Ebaf observed in C57BL/6J Cited $2^{-1-}$ embryos.

One mechanism explaining these results is that CITED2, a transcriptional coactivator for TFAP2 and LIM-domain transcription factors $^{9,15,16}$, may coactivate Nodal-activated gene transcription in the left lateral plate mesoderm. In support of this idea, in the case of Pitx2c, we showed that endogenous CITED2 and TFAP2 can be detected at the Pit $2 c$ P1 promoter in embryonic hearts and $\mathrm{C} 2 \mathrm{C} 12$ cells. We also showed that acetylated histone $\mathrm{H} 3$ is present at the Pitx $2 c$ P 1 promoter, indicating that it is in a transcriptionally active state. In addition, we showed that the Pitx $2 c$ P1 promoter contains evolutionarily conserved TFAP2 binding sites, that sequences from the Pitx2c P1 promoter directly bind TFAP2 in vitro and that TFAP2 activates and CITED2 coactivates the Pit $2 c$ P 1 promoter in transfection assays. Our experiments also identify certain TFAP2 binding sites in the Pitx2c P1 promoter that are necessary for TFAP2 and CITED2-mediated activation. These results do not however exclude the possibility that endogenous CITED2 or TFAP2 윽 may be recruited to the Pitx $2 c$ P1 promoter independently of these TFAP2 binding sites, for example, through Sp1 or LIM-domain transcription factors ${ }^{16,38}$.

Our results also show that the mouse and human Pitx2c P1 promoters are highly conserved, with large blocks of sequence identity of $85 \%$ or more. Genome-wide comparisons between human and mouse indicate that the average sequence identity is $69 \%$ in introns, $75 \%$ in untranslated regions and $85 \%$ in coding regions ${ }^{39}$. The high degree of sequence conservation of the Pit $2 c$ P1 promoter suggests that its functions have been evolutionarily conserved under natural selection over $\sim 75$ million years of mammalian species divergence. This is probably important, as the characteristic features of the mammalian heart (complete atrial, ventricular and outflow tract septation, and left-sidedness of the aortic arches ${ }^{40}$ ) are controlled by Pitx $2 c^{25}$.

In conclusion, these results establish new roles for Cited 2 in controlling left-right patterning and Nodal-activated gene transcription. They show that the cardiovascular and laterality defects in Cited $2^{-l-}$ embryos can be explained by a deficiency of the NodalPitx $2 c$ pathway, providing a unifying mechanism for the apparently unrelated malformations of the cardiac septum, outflow tract and aortic arch previously observed in Cited2-deficient mice. These cardiovascular malformations were hitherto not recognized as laterality defects. Our results indicate the existence of genetic modifiers that affect the development of overt laterality defects such as isomerism. Moreover, they show that Pitx $2 c$ is required for the development of the characteristic features of the mammalian heart and suggest that the control of Pitx $2 c$ expression is conserved through mammalian evolution. Our results imply that common congenital heart defects, such as cardiac septal, outflow tract and aortic arch malformations ${ }^{41}$, may be the sole manifestation of a laterality defect and suggest that mutations in genes controlling laterality may result in common forms of congenital heart disease.

\section{METHODS}

Embryos and MRI. We backcrossed Cited $2^{+/-}$mice (Cited $2^{\text {tmBhal }}, 129 \mathrm{P} 2 \mathrm{OlaHsd}$ $\left.\times \mathrm{C} 57 \mathrm{BL} / 6 \mathrm{~J}^{9}\right)$ to $\mathrm{C} 57 \mathrm{BL} / 6 \mathrm{~J}$ and to $129 \mathrm{~Sv}$ mice for nine generations to generate coisogenic mice. Pit $\times 2 c^{+/-}$mice were described previously ${ }^{25}$. We collected embryos at the indicated time points after detection of a vaginal plug $(0.5$ d.p.c.) and genotyped them using allele-specific PCR (primer details available on request). We carried out MRI and data analysis as described previously ${ }^{13}$, with a final image resolution of either $12.7 \times 12.7 \times 19.5 \mu \mathrm{m}$ per voxel or $25.4 \times 25.4 \times 24.4 \mu \mathrm{m}$ per voxel $(x, y$ and $z$ axes). All animal experimentation was done under UK Home Office authorization and regulations.

In situ hybridization. We carried out in situ hybridization essentially as described $^{42}$ using digoxigenin-UTP-labeled riboprobes (Roche). The Hand1 probe was a gift from P. Riley (ICH, London) and the Pitx2 probe was described previously ${ }^{43}$. Nodal, Leftb, Ebaf and $C f c 1$ probes were gifts from H. Hamada (Osaka University, Japan).

DNA sequence analysis. We identified transcription factor binding sites using DNAStar software and SignalScan TFD ${ }^{33}$. The consensus TFAP2 binding sites are S00346, YCSCCMNSSS; S01544, GSSWGSCC; and S01936, CCCMNSSS. We carried out BLAST analysis as described ${ }^{44}$. The mouse and human Pitx2c P1 promoter sequences correspond to Ensembl mouse chromosome 3 132825298132826982 and human chromosome 4 112004151-112002466.

Plasmids. CMV-CITED2, CMV-TFAP2A, CMV-TFAP2C, CMV-luciferase and CMV-lacZ were described previously ${ }^{5,9}$. We constructed plasmids P1-Luc-ASE and P1-Luc by subcloning appropriate fragments from plasmid 0.9-P1 (gift from H. Hamada and H. Shiratori ${ }^{32}$, Osaka University, Japan) into pGL3Basic (Promega) using standard techniques ${ }^{45}$ (Supplementary Methods online). We mutated the TFAP2 binding sites in oligonucleotides IJ and QR and the second TFAP2 binding site in oligonucleotide MN (the first site did not bind TFAP2 in vitro) using the QuikChange Site-Directed Mutagenesis Kit (Stratagene) and confirmed them by sequencing. Oligonucleotide and plasmid details are available on request.

Antibodies. We obtained affinity-purified monoclonal antibodies specific to CITED2 and Stat1 from Novus Biologicals ${ }^{5}$ (JA22, IgG1) and from Santa Cruz Biotechnology (C-136, sc-464X, IgG1). TFAP2A-specific rabbit polyclonal antibody HCH16 and preimmune serum were gifts from H. Hurst (CRUK, London). We obtained polyclonal antibodies to TFAP2A (C-18, sc-184X), TFAP2C (H-77, sc-8977X), Stat2 (C-20, sc-476X) and Flag (D-8, sc-807) from Santa Cruz Biotechnology. We obtained antibody to acetylated histone H3 (06-599) from Upstate Cell Signalling Solutions and polyclonal antibody to mouse IgG (Z0109) from DAKO.

Cells and transfections. We obtained $\mathrm{C} 2 \mathrm{C} 12$ and Hep3B cells from ATCC. We transfected cells and measured luciferase and $\beta$-galactosidase activities the next day as described ${ }^{15}$. We assessed statistical significance using the $t$-test, using $P \leq 0.05$ as statistically significant.

ChIP assays and EMSA. We carried out ChIP essentially as described ${ }^{46}$ from C2C12 cells or hearts isolated from embryos at 13.5 d.p.c. using the indicated primary antibodies and a secondary polyclonal antibody to mouse IgG for primary monoclonal antibodies (Supplementary Methods online). We carried out EMSA as described ${ }^{15}$. MTIIa is a wild-type TFAP2 binding site from the metallothionein IIa gene promoter. MTIIaMUT contains a mutation that abolishes binding, and PPAR contains a PPAR $\gamma$ binding site. Oligonucleotide sequences are available on request.

URLs. SignalScan TFD is available at http://bimas.cit.nih.gov/molbio/. 2-Sequence BLAST is available at http://www.ncbi.nlm.nih.gov/blast/bl2seq/ bl2.html.

Note: Supplementary information is available on the Nature Genetics website.

\section{ACKNOWLEDGMENTS}

We thank H. Hamada, H. Shiratori, H. Hurst and P. Riley for reagents; H. Koseki and Y. Fujimura for ChIP protocols; and R. Copley for discussions. C.R.F. is a Wellcome Trust Prize Student, and S.B. a Wellcome Trust Senior Research Fellow. These studies were funded by the Wellcome Trust.

\section{COMPETING INTERESTS STATEMENT}

The authors declare that they have no competing financial interests. 
Received 18 August; accepted 7 September 2004

Published online at http://www.nature.com/naturegenetics/

1. Bruneau, B.G. Transcriptional regulation of vertebrate cardiac morphogenesis. Circ Res. 90, 509-519 (2002).

2. Goodman, R.H. \& Smolik, S. CBP/p300 in cell growth, transformation, and development. Genes Dev. 14, 1553-1577 (2000).

3. Petrij, F. et al. Rubinstein-Taybi syndrome caused by mutations in the transcriptional co-activator CBP. Nature 376, 348-351 (1995).

4. Stevens, C.A. \& Bhakta, M.G. Cardiac abnormalities in the Rubinstein-Taybi syndrome. Am. J. Med. Genet. 59, 346-348 (1995).

5. Bhattacharya, S. et al. Functional role of p35srj, a novel p300/CBP binding protein, during transactivation by HIF-1. Genes Dev. 13, 64-75 (1999).

6. Dunwoodie, S.L., Rodriguez, T.A. \& Beddington, R.S.P. Msg1 and Mrg1, founding members of a gene family, show distinct patterns of gene, expression during mouse embryogenesis. Mech. Dev. 72, 27-40 (1998)

7. Sun, H.B., Zhu, Y.X., Yin, T., Sledge, G. \& Yang, Y.C. MRG1, the product of a melanocyte-specific gene related gene, is a cytokine-inducible transcription factor with transformation activity. Proc. Natl. Acad. Sci. USA 95, 13555-13560 (1998).

8. Freedman, S.J. et al. Structural basis for negative regulation of hypoxia-inducible factor-1 $\alpha$ by CITED2. Nat. Struct. Biol. 10, 504-512 (2003).

9. Bamforth, S.D. et al. Cardiac malformations, adrenal agenesis, neural crest defects and exencephaly in mice lacking Cited2, a new Tfap2 co-activator. Nat. Genet. 29, 469474 (2001).

10. Barbera, J.P. et al. Folic acid prevents exencephaly in Cited2 deficient mice. Hum. Mol. Genet. 11, 283-293 (2002).

11. Weninger, W.J. \& Mohun, T. Phenotyping transgenic embryos: a rapid 3-D screening method based on episcopic fluorescence image capturing. Nat. Genet. 30, 59-65 (2002).

12. Yin, Z. et al. The essential role of Cited2, a negative regulator for $\mathrm{HIF}-1 \alpha$, in heart development and neurulation. Proc. Natl. Acad. Sci. USA 99, 10488-10493 (2002).

13. Schneider, J.E. et al. Rapid identification and $3 D$ reconstruction of complex cardiac malformations in transgenic mouse embryos using fast gradient echo sequence magnetic resonance imaging. J. Mol. Cell. Cardiol. 35, 217-222 (2003).

14. Kranc, K.R. et al. Transcriptional Coactivator Cited2 Induces Bmi1 and Mel18 and Controls Fibroblast Proliferation via Ink4a/ARF. Mol. Cell. Biol. 23, 7658-7666 (2003)

15. Braganca, J. et al. Physical and Functional Interactions among AP-2 Transcription Factors, p300/CREB-binding Protein, and CITED2. J. Biol. Chem. 278, 1602116029 (2003)

16. Glenn, D.J. \& Maurer, R.A. MRG1 Binds to the LIM Domain of Lhx2 and may function as a coactivator to stimulate glycoprotein hormone alpha-subunit gene expression. J. Biol. Chem. 274, 36159-36167 (1999).

17. Brewer, $S$., Jiang, $X$, Donaldson, $S$., Williams, T \& Sucov, H.M. Requirement for AP-2 $\alpha$ in cardiac outflow tract morphogenesis. Mech. Dev. 110, 139-149 (2002).

18. Satoda, M. et al. Mutations in TFAP2B cause Char syndrome, a familial form of patent ductus arteriosus. Nat. Genet. 25, 42-46 (2000).

19. Thomas, T., Yamagishi, H., Overbeek, P.A., Olson, E.N. \& Srivastava, D. The bHLH factors, dHAND and eHAND, specify pulmonary and systemic cardiac ventricles independent of left-right sidedness. Dev. Biol. 196, 228-236 (1998).

20. Gage, P.J., Suh, H. \& Camper, S.A. Dosage requirement of Pitx2 for development of multiple organs. Development 126, 4643-4651 (1999).

21. Kitamura, K. et al. Mouse Pitx2 deficiency leads to anomalies of the ventral body wall, heart, extra- and periocular mesoderm and right pulmonary isomerism. Development 126, 5749-5758 (1999)

22. Lin, C.R. et al. Pitx2 regulates lung asymmetry cardiac positioning and pituitary and tooth morphogenesis. Nature 401, 279-282 (1999).

23. Lu, M.F., Pressman, C., Dyer, R., Johnson, R.L. \& Martin, J.F. Function of Rieger syndrome gene in left-right asymmetry and craniofacial development. Nature 401 276-278 (1999).
24. Liu, C., Liu, W., Lu, M.F., Brown, N.A. \& Martin, J.F. Regulation of left-right asymmetry by thresholds of Pitx2c activity. Development 128, 2039-2048 (2001).

25. Liu, C. et al. Pitx2c patterns anterior myocardium and aortic arch vessels and is required for local cell movement into atrioventricular cushions. Development 129 , 5081-5091 (2002)

26. Hamada, H., Meno, C., Watanabe, D. \& Saijoh, Y. Establishment of vertebrate left-right asymmetry. Nat. Rev. Genet. 3, 103-113 (2002).

27. Saijoh, Y., Oki, S., Ohishi, S. \& Hamada, H. Left-right patterning of the mouse lateral plate requires nodal produced in the node. Dev. Biol. 256, 160-172 (2003).

28. Meno, C et al. Diffusion of nodal signaling activity in the absence of the feedback inhibitor Lefty2. Dev. Cell 1, 127-138 (2001).

29. Saijoh, Y. et al. Left-right asymmetric expression of lefty2 and nodal is induced by a signaling pathway that includes the transcription factor FAST2. Mol. Cell 5, 35-47 (2000).

30. Shen, M.M., Wang, H. \& Leder, P. A differential display strategy identifies Cryptic, a novel EGF-related gene expressed in the axial and lateral mesoderm during mouse gastrulation. Development 124, 429-442 (1997).

31. Yamamoto, M. et al. Nodal signaling induces the midline barrier by activating Nodal expression in the lateral plate. Development 130, 1795-1804 (2003).

32. Shiratori, $\mathrm{H}$. et al. Two-step regulation of left-right asymmetric expression of Pitx2: initiation by nodal signaling and maintenance by Nkx2. Mol. Cell 7, 137-149 (2001).

33. Prestridge, D.S. SIGNAL SCAN: a computer program that scans DNA sequences for eukaryotic transcriptional elements. Comput. Appl. Biosci. 7, 203-206 (1991).

34. Brennan, J., Norris, D.P. \& Robertson, E.J. Nodal activity in the node governs left-right asymmetry. Genes Dev. 16, 2339-2344 (2002).

35. Norris, D.P., Brennan, J., Bikoff, E.K. \& Robertson, E.J. The Foxh1-dependent autoregulatory enhancer controls the level of Nodal signals in the mouse embryo. Development 129, 3455-3468 (2002).

36. Yan, Y.T. et al. Conserved requirement for EGF-CFC genes in vertebrate left-right axis formation. Genes Dev. 13, 2527-2537 (1999).

37. Gaio, U. et al. A role of the cryptic gene in the correct establishment of the left-right axis. Curr. Biol. 9, 1339-1342 (1999).

38. Pena, P. et al. Activator protein-2 mediates transcriptional activation of the CYP $11 \mathrm{~A} 1$ gene by interaction with Spl rather than binding to DNA. Mol. Endocrinol. 13, 1402-1416 (1999)

39. Waterston, R.H. et al. Initial sequencing and comparative analysis of the mouse genome. Nature 420, 520-562 (2002).

40. Kent, G.C. \& Carr, R.K. Comparative Anatomy of the Vertebrates 314-350 (McGraw Hill, Boston, 2001).

41. Clark, E.B. Etiology of congenital cardiac malformations: epidemiology and genetics. in Moss and Adams' Heart Disease in Infants, Children, and Adolescents (eds. Allen, H.D., Gutgessell, H.P., Clark, E.B. \& Driscoll, D.J.) 64-79 (Lipincott Williams \& Wilkins, Philadelphia, 2001).

42. Wilkinson, D.G. Whole mount in situ hybridization of vertebrate embryos. in In situ Hybridization (ed. Wilkinson, D.G.) 75-83 (IRL, Oxford, 1992).

43. Ryan, A.K. et al. Pitx2 determines left-right asymmetry of internal organs in vertebrates. Nature 394, 545-551 (1998).

44. Tatusova, T.A. \& Madden, T.L. BLAST 2 Sequences, a new tool for comparing protein and nucleotide sequences. FEMS Microbiol. Lett. 174, 247-250 (1999).

45. Ausubel, F. et al. Short Protocols in Molecular Biology (John Wiley \& Sons, 1995).

46. Boyd, K.E., Wells, J., Gutman, J., Bartley, S.M. \& Farnham, P.J. c-Myc target gene specificity is determined by a post-DNA binding mechanism. Proc. Natl. Acad. Sci. USA 95, 13887-13892 (1998).

47. Brown, N.A. \& Anderson, R.H. Symmetry and laterality in the human heart: developmental implications. in Heart Development (eds. Harvey, R.P. \& Rosenthal, A.) 447-462 (Academic, San Diego, 1999).

48. Kaufman, M. \& Bard, J.B.L. The Anatomical Basis of Mouse Development 33-46 (Academic, San Diego, 1999). 